\title{
Anja Becker u. Jan Mohr (Hgg.): Alterität als Leitkonzept für historisches Interpretieren
}

\author{
Benz, Maximilian
}

DOI: https://doi.org/10.1515/bgsl-2014-0006

Posted at the Zurich Open Repository and Archive, University of Zurich ZORA URL: https://doi.org/10.5167/uzh-154067

Journal Article

Published Version

Originally published at:

Benz, Maximilian (2014). Anja Becker u. Jan Mohr (Hgg.): Alterität als Leitkonzept für historisches Interpretieren. Beiträge zur Geschichte der deutschen Sprache und Literatur, 136(1):137-172.

DOI: https://doi.org/10.1515/bgsl-2014-0006 


\section{Besprechungen}

Anja Becker u. Jan Mohr (Hgg.): Alterität als Leitkonzept für historisches Interpretieren, Berlin: Akademie Verlag 2012, 436 S. (Deutsche Literatur. Studien und Quellen 8)

Eines wird man den Herausgebern dieses Bandes keinesfalls vorwerfen können: Tiefstapelei. Bereits der Titel deutet nicht nur die prätendierte Reichweite der Unternehmung Anja Beckers und Jan Mohrs an, sondern enthält auch eine gewagte These, denn in ihm werden Zentralbegriffe mediävistischer Literaturwissenschaft auf kühne Weise miteinander verbunden: Das ganz grundsätzliche, in seinen Implikationen aber umstrittene Problem der >Alterität` wird zum Leitkonzept für das vorgeschlagen, was nach wie vor das Hauptgeschäft der älteren deutschen Philologie ist: für das historische Interpretieren. Die Herausgeber möchten »die Reflexion über die Kategorie >Alterität` wieder auf eine explizit programmatische Ebene heben und sie so im Theorie- und Methodendiskurs der philologischen Disziplinen (re)installieren« (S. 6). Dieses Vorhaben muss überraschen: Denn nach einigen Exzessen der späten 1980er und der 1990er Jahre, in denen Alterität, wie Susanne Köbele im vorliegenden Band schreibt: »in eine sinnlose, denkunmögliche Absolutheit« (S. 303) hineingetrieben wurde, wird in jüngster Zeit die »kulturell-historische (vertikale) Distanz zwischen Mittelalter und Moderne ${ }^{1}$ im Kontext spezifischer methodologischer Reflexionen differenziert bearbeitet. Während etwa Ursula Peters die Diskussionen um die Alterität mittelalterlicher Literatur in Hinsicht auf Chancen einer stärker materialphilologischen Ausrichtung perspektiviert, ${ }^{2}$ schlägt Christian Kiening eine Differenzierung zwischen Alterität im Plural und Alterität im Singular vor. ${ }^{3}$ Im Zuge dieser Modifikationen ist die Diskussion um das Problem >der Alterität an einen gewissen Endpunkt geraten, so dass Manuel Braun im Jahre 2008 mit einigem Recht in einem >Call for papers dazu einlud, auf einer Tagung des Brackweder

1 Peter Strohschneider: [Art.] Alterität, in: RLW, Bd. 1, 1997, S. 58-59, hier S. 58.

2 Ursula Peters: >Texte vor der Literatur ? Zur Problematik neuerer Alteritätsparadigmen der Mittelalter-Philologie, in: Poetica 39 (2007), S. 59-88.

3 Christian Kiening: Alterität und Methode. Begründungsmöglichkeiten fachlicher Identität, in: Mitteilungen des Deutschen Germanistenverbandes 52 (2005), S. 150-166, hier S. 162 (Herv. i. O.): »Zum einen also: Alterität im Plural, als relationale und deskriptive Kategorie, die hinsichtlich des beobachteten Systems Differenzierungen ermöglicht - komplexere `Beschreibungen` mittelalterlicher Texte als von Heteronomie, Variation und Präsenz geprägten. Zum anderen: Alterität im Singular, als systematische und methodologische Kategorie, die hinsichtlich des Beobachtungssystems Differenzierungen erlaubt - komplexere `Modelle` mittelalterlicher Textualität im Spannungsfeld von Materialität und Medialität.« 
Arbeitskreises das Konzept der Alterität auf seine Grenzen hin zu befragen und die Möglichkeiten eines Weiterdenkens anzudeuten, dabei jedenfalls immer von Alterität auf der Metaebene auszugehen. ${ }^{4}$

Demgegenüber bringen Anja B e c k e r und Jan M o h r (S. 1-58) die Kategorie der Alterität in umfassenderem Sinne wieder ins Spiel, indem sie die vertikale mit der horizontalen Differenz verbinden (was auch Folgen für die Länge des, zurückhaltend formuliert, einlässlichen Forschungsberichts hat). Becker und Mohr rekurrieren - zunächst heuristisch - auf die Ergebnisse der Fremdeforschung, mithin auf Bernhard Waldenfels’>Phänomenologie des Fremden`. Sie schlagen vor, dass Alterität sowohl eine relationale Beziehungskonstellation als auch prinzipielle Unverfügbarkeit meine (vgl. S. 41-43). Alterität werde nicht von einem unbeteiligten Dritten ausgewiesen: Der Interpretierende spreche $»$ nicht nur aus der Beobachter-, sondern zugleich aus der Mitspielerperspektive« (S. 42) und sei von seiner eigenen Unterscheidung betroffen.

Auch wenn die Herausgeber aus dem Entwickelten lediglich ableiten, dass das »Verhältnis von Relationalität und Unverfügbarkeit auf eine methodologische Klärung der Stellung des Interpreten zu seinen Gegenständen« (S. 43) dränge, führt die Einleitung letztlich zum Konzept responsiven Interpretierens, das aber nur Anja B e c k e r (S. 73-101) in ihrem eigenen Beitrag im Anschluss an die von Bernhard Waldenfels (S. 61-71) prägnant vorgestellte responsive Phänomenologie des Fremden entwickelt. »Anders als die geläufige intentionale Phänomenologie«, so Waldenfels,

"geht die responsive Phänomenologie von dem aus, was uns auffällt, einfällt, was uns erstaunt, erschreckt oder verletzt. Sie setzt an bei einem `Pathos`, einem Widerfahrnis, einem Af-fekt [sic!], bei dem also, ১wovon` wir getroffen, angerührt, angesprochen sind und das nur indirekt und nachträglich in der `Response` fassbar ist als das, `worauf $\triangleleft$ wir antworten« (S. 63).

Von zentraler Bedeutung ist für Waldenfels die Diastase, der »Hiatus zwischen dem vorgängigen Pathos und der nachträglichen Response« (S. 64). Daran anschließend, kann Becker zeigen, wie sich die responsive Phänomenologie mit dem entwickelten komplexen Alteritätsverständnis verbinden lässt. Der Vorschlag verdient ein längeres Zitat:

»Responsives Interpretieren bricht die scheinbare Aporie der Alteritätsdiskussion auf, in Folge derer die mittelalterliche Literatur einmal zum unzugänglichen Anderen, einmal zur Vorform von Modernität erklärt wird. [...] Es wird also nicht der Anschein erweckt, als

4 Manuel Braun (Hg.): Wie anders war das Mittelalter? Fragen an das Konzept der Alterität, Göttingen 2013 (Aventiuren 9). 
stünde man gleichsam wie ein unbeteiligter Dritter über der Epochengrenze und vergliche beide Seiten objektiv. Dagegen wird der diastatische Prozess einer gleichzeitigen Eingrenzung meines Erfahrungsbereiches und Ausgrenzung eines fremden Erfahrungsraumes betont, in welchem beide Bereiche zuallererst entstehen. [...] Denn jede Lektüre-Erfahrung, egal ob ein Literaturprofessor oder ein Literaturliebhaber sie erlebt, ist eine subjektive. Diese kann sich einstellen, wenn man einfach snur liest, oder wenn sich beim methodischen Lesen plötzlich ein Spalt auftut, durch den hindurch Ungewohntes und Unvorhergesehenes uns trifft. Ein responsives Interpretieren sucht solche Erfahrungen auf, in denen sich ein Anspruch eines radikal Fremden äußert, im vollen Bewusstsein, dass man solche Erfahrungen nicht herstellen kann, sondern sie uns >überkommen« (S. 89 ff.).

Dass hier allerdings der Unterschied zwischen einem philologisch geschulten Blick auf Literatur und einer Laienrezeption dezidiert eingeebnet wird, zeigt, dass es eher um eine Rezeptions- als um eine Interpretationstheorie geht und dass somit responsives Interpretieren, oder, wie ich es nennen würde, eine responsive Lektürepraxis keineswegs geeignet erscheint, das Problem historischen Interpretierens $\mathrm{zu}$ adressieren. Sowohl Waldenfels als auch Becker heben hervor, dass eine responsive Lektürepraxis keinen Rückfall hinter jede philologische Methodik darstelle (vgl. etwa S. 65 oder S. 93). Da sie allerdings auch die Unverfügbarkeit der Widerfahrnisse betonen, wäre noch ausführlicher zu begründen, in welchem Verhältnis Affekt und Methode stehen: So lässt sich anhand von Beckers responsiver Lektüre des >Wigalois<-Prologs in der Leidener Handschrift fragen, was genau in methodischer und in historischer Hinsicht aus der referentiellen Verwirrung abzuleiten ist, die durch das heute gerade unübliche laute Lesen eines Rollendeiktikons entsteht (vgl. S. 98). Trotz dieser Einwände darf nicht übersehen werden, dass eine responsive Lektürepraxis in vielerlei Hinsicht auch für literaturwissenschaftliche Zusammenhänge interessant sein kann; so mag etwa die phänomenologische Reduktion zur Offenlegung und Kritik eigener oder fremder Präsuppositionen beitragen (vgl. S. 94). Nur scheint mir das mehr oder minder explizite Versprechen, mit einem von der responsiven Phänomenologie ausgehenden neuen Alteritätsverständnis auch ein >Leitı-Konzept für historisches Interpretieren zu gewinnen, nicht eingelöst zu werden. Demgegenüber gibt es in der mediävistischen Literaturwissenschaft eine Vielfalt an Heuristiken und Konzepten, die in je spezifischer Weise die Alteritätsproblematik differenziert bearbeiten, wie die einzelnen Beiträge eindrücklich zeigen.

So betont Burkhard H a s e b r i n k (S. 103-117) zu Beginn seines Beitrages zwar die »methodologische Sprengkraft« (S. 105) des Becker'schen Vorschlags, liest Meister Eckharts >Buch der göttlichen Tröstung` dann allerdings keineswegs responsiv und klärt auch nicht seine Stellung zum Untersuchungsgegenstand, sondern rekonstruiert die für die mittelalterliche Mystik zentrale Figur des Selbstverlustes: "Nur in der Aufgabe des Gottesbezugs, im Lassen Gottes, kann sich Gott im Innersten des gelassenen Menschen in seiner selbstbezüglichen Seinsweise, nicht in der Art 
eines Gehabtseins, sausbreiten«" (S. 111). Zwar formuliert Hasebrink Berührungspunkte zwischen Andersheit und Einheit, wie sie bei Meister Eckhart gedacht werden, und einer Phänomenologie des Fremden (vgl. bes. S. 116 f.), doch in Hinsicht auf seinen eigenen Text verfährt er - sit venia verbo! - hermeneutisch und verfasst so eine luzide, gemäß Kohäsionsprinzip gegenstandsadäquate Interpretation. ${ }^{5}$

Hasebrinks Beitrag ist gleichwohl der, der dem Becker'schen Vorschlag noch am nächsten steht. Michael W a lt e n berger (S. 121-140) hingegen eröffnet seine Analyse des ersten stratado des >Lazarillo de Tormes Widerspruch zur Methode des responsiven Interpretierens:

»Vielleicht ist es also günstiger, nicht bei vermeintlichen Evidenzen einer unmittelbaren Erfahrung von Fremdheit am Text anzufangen, sondern bei der hypothetischen, kontextuell plausibilisierten (und revidierbaren) initialen Setzung einer Alteritätsdifferenz zwischen dem kulturellen Raum des Beobachters und einem davon epochal unterschiedenen kulturellen Raum des Textes« (S. 126).

Dabei gelingt Waltenberger $u$. a. unter Rekurs auf Gilles Deleuzes >Differenz und Wiederholung ‘ die gegenüber modernem Erzählen alteritäre, serielle Erzählstruktur zu beschreiben und die Annahme »einer Einheit des textuellen Ichs« (S. 139) zurückzuweisen.

Es ist bemerkenswert, dass sich auch der Mitherausgeber Jan M o h r (S. 141169) in seinem Beitrag zu Identitätskonzepten in Hartmanns >Iwein` dezidiert von der Waldenfels'schen >Phänomenologie des Fremden ‘ abwendet (S. 150) und stattdessen, den Vorschlägen Christian Kienings folgend (S. 147, S. 167), anhand einer genauen Lektüre zeigt, dass im Hartmann'schen Text zwar ein Konzept personaler Identität zu greifen sei; Iweins Selbstadressierungen bezögen sich aber auf ein ısprachpragmatisches`, nicht auf ein sepistemisches Ich`(S. 145, S. 166). Mohr setzt sich hierbei kritisch mit dem Konzept einer spartizipativen Identität` (Alois Hahn, vgl. S. 159) auseinander und überprüft dessen Implikationen.

Auch Susanne Köbele (S. 303-333) beschreitet den in der Einleitung vorgeschlagenen Weg nicht (vgl. S. 304), sondern nähert sich dem Problem der Alterität, für sie ein »dialektischer Prozessbegriff« (S. 304, vgl. auch bes. S. 331), am konkreten historischen Gegenstand und seiner Forschungsgeschichte. Durch eine genaue Analyse der Implikationen von Reimbrechungen und >Bildbrüchen entdeckt Köbele in Konrads `Goldener Schmiede` eine »Aufmerksamkeitskonkurrenz von Klang und Sinn« (S. 313), die vom Rezipienten eine schwebende Aufmerksamkeit verlange; in methodischer Hinsicht weist sie auf die Interferenzen

5 Vgl. Andreas Kablitz: Theorie der Literatur und Kunst der Interpretation. Zu einigen Blindstellen literaturwissenschaftlicher Theoriebildung, in: Poetica 41 (2009), S. 219-231. 
»zwischen sex post rekonstruierter (retrospektiver) und sim Text konstruierter (kalkulierter) Alterität« (S. 329) hin - ein spezifisch literaturwissenschaftliches Problem (S. 331)!

Es zeigt sich somit in aller Deutlichkeit, dass die Idee, die dem gesamten Vorhaben vorausgeht (Vorwort und S. 99), nämlich die Befruchtung der Alteritätsdiskussion durch einen Rekurs auf die Waldenfels'sche >Phänomenologie des Fremden`, sich im Lauf der Tagung nicht als gute herausgestellt hat. Den vorliegenden Sammelband zeichnet eine Disparität, ja innere Widersprüchlichkeit aus, auch wenn an mehreren Stellen (etwa in den Beiträgen von Bernhard Waldenfels und Karl-Siegbert Rehberg) versucht wird, Kohärenz herzustellen. Die These, auf die die gesamte Einleitung zuläuft, und damit das Surplus gegenüber dem bisherigen Forschungsstand (vgl. schon S. 11), nimmt nur in den Beiträgen von Waldenfels und Becker selbst konkrete Gestalt an und wird nicht wirklich auf ihre Leistungsfähigkeit hin überprüft, sondern - sofern überhaupt auf sie nennenswert eingegangen wird - mehr oder weniger explizit zurückgewiesen.

Ich beklage dies keineswegs. Denn die im vorliegenden Band dokumentierte Diskussion ist durchaus zielführend; allerdings hätten die Auseinandersetzungen in der Einleitung offensiv angesprochen (vgl. hingegen die bloßen Andeutungen etwa auf S. 45) und Rückschlüsse auf die Konzeption des Bandes gezogen werden müssen. Dass dies versäumt wurde, ist umso bedauerlicher, als die Beiträge ausnahmslos etwas zur Diskussion über die Alteritätsproblematik beitragen und vielschichtige, differenzierte, stets lesenswerte Einblicke in verschiedene wichtige Forschungsfelder der Altgermanistik und benachbarter Disziplinen liefern (vgl. neben dem genuin musikwissenschaftlichen Beitrag Lorenz Welkers [S. 243-261] auch den von Volker M e r t e n s [S. 335-363] und den theaterwissenschaftlichen von Julia S t e n z e 1 [S. 365-386] ${ }^{6}$ ).

Nur hinweisen kann ich im Folgenden auf verschiedene, je sehr anschlussfähige Studien, so auf Rabea Kohnen (S. 171-195) spannenden, in medialer Hinsicht differenzierten Beitrag über das salternate ending` der Druckfassung von ১Salman und Morolf`, die 1499 in der Offizin von Matthias Hupfuff gedruckt wurde. Kohnen macht Konzepte und Begriffe der Filmwissenschaft für das Verständnis eines Textschlusses fruchtbar, dem man zuvor meist mit Unverständnis gegenübertrat. Jan-Dirk M ü 11 e r (S. 263-284) untersucht das `Donaueschinger Passionsspiel«; die genaue Analyse des Texts und seines Kontexts führt ihn zu einer komplexen Modellierung des Verhältnisses von Repräsentation und Präsenz und einer

6 Gerade für Stenzels eigene theoretische Überlegungen wäre ein Rekurs auf das Transformationskonzept hilfreich gewesen; vgl. Hartmut Böhme [u. a.] (Hgg.): Transformation. Ein Konzept zur Erforschung kulturellen Wandels, München 2011. 
diskussionswürdigen Modifizierung des Fiktionskonzepts, die zwischen dem >AlsOb der theatralen Inszenierung und der im Geschehen dargestellten Heilswahrheit differenziert. Tobias B ulang s (S. 285-302) historisch informierte Untersuchung der Sprache Fischarts zeigt, dass

»Fischarts Vorgehen einer möglichst vollständigen Versammlung von Wörtern $\mathrm{zu}$ allen möglichen Sachgruppen und die Arbeit an eigens kreierten Benennungsalternativen als nicht unbedeutendes, jedoch höchst eigenwilliges linguistisches Projekt zu betrachten« (S. 299)

ist. Bulang demonstriert ebenso wie Andreas K a b lit z (S. 199-242) die Notwendigkeit historischer Kontextmodellierung. Kablitz rekonstruiert die komplexen Zusammenhänge zwischen Alterität und Ästhetik - beide Konzepte stammen nicht zufällig aus der Zeit um 1800 - im Rahmen einer Analyse der Auerbach'schen Deutung des zehnten Gesangs des >Inferno ${ }^{2}$ aus Dantes >Divina Commedia ${ }^{7}$ und setzt dieser eine eigene Interpretation entgegen. Was die von Kablitz analysierten historischen Bedingungen der Wahrnehmung von Alterität betrifft, zeigt Peter S t r o h s c h n e i d e r (S. 378-416) ausgehend von Reinhart Kosellecks asymmetrischen Gegenbegriffen mit Fokus auf Michel de Montaigne, wie in der Frühen Neuzeit Negierungsverzichte zu Unbekanntheitsgewinnen führen; im 18. Jahrhundert schließlich etabliere sich mit dem plurale tantum >Kultur »eine Praxis des Vergleichens von Ungleichen, die auf Axiologie und Normativität geradezu programmatisch verzichten will« (S. 413).

Wie lässt sich dies alles nun bilanzieren? Neben einer gewissen Ratlosigkeit gegenüber dem Gesamtkonzept des Bandes bleibt am Schluss nicht nur die Erkenntnis, auf welch vielfältige und differenzierte Weisen die Probleme der ıkulturell-historischen Distanz zwischen Mittelalter und Moderne`, die sich je nach Gegenstand unterschiedlich darstellen, unter Heranziehung spezifischer Heuristiken, Modelle und Methoden in Einzelstudien ${ }^{8}$ bearbeitet wurden, sondern auch die Einsicht, dass die vorgeschlagene responsive Lektürepraxis noch einer eingehenderen, kritisch prüfenden Anwendung harrt.

Dr. Maximilian Benz: Universität Zürich, Deutsches Seminar, Schönberggasse 9, CH-8001 Zürich, E-Mail: Maximilian.Benz@ds.uzh.ch

7 Vgl. in diesem Zusammenhang bes. auch die Deutung eines Jacob-Burckhardt-Zitats auf S. 240. 8 Andreas Kablitz zeigt schlagend, warum dies so ist; vgl. bes. S. 214: „Universalismus oder Alterität in der Geschichte nicht anders als Autonomie oder Widerspiegelung in der Kunst bilden jeweils Grenzwerte einer Skala, die ein Mehr oder Minder durchaus zulässt. Es handelt sich dabei zugleich, auch dies ist eine Konsequenz der hier vorgeschlagenen Verwandlung klassifikatorischer in komparative Begriffe, um nicht mehr als um heuristische Fragen, über die fallweise und das heißt: nur empirisch zu entscheiden ist." 